\title{
Metabolic characteristics of solid pseudopapillary neoplasms of the pancreas: their relationships with high intensity ${ }^{18}$ F-FDG PET images
}

\author{
Minhee Park ${ }^{1, *}$, Ho Kyoung Hwang ${ }^{2,4, *}$, Mijin Yun ${ }^{3,4}$, Woo Jung Lee ${ }^{2,4}$, Hoguen Kim ${ }^{1}$ \\ and Chang Moo Kang ${ }^{2,4}$ \\ ${ }^{1}$ Departments of Pathology and BK21 PLUS for Medical Science, Yonsei University College of Medicine, Seoul, Korea \\ ${ }^{2}$ Department of Hepatobiliary and Pancreatic Surgery, Department of Surgery, Yonsei University College of Medicine, Seoul, Korea \\ ${ }^{3}$ Department of Nuclear Medicine, Yonsei University College of Medicine, Seoul, Korea \\ ${ }^{4}$ Pancreatobiliary Cancer Clinic, Yonsei Cancer Center, Severance Hospital, Seoul, Korea \\ *These authors contributed equally to this work \\ Correspondence to: Hoguen Kim, email: hkyonsei@yuhs.ac \\ Chang Moo Kang, email: cmkang@yuhs.ac
}

Keywords: solid pseudopapillary neoplasm; metabolomics; metabolism; ${ }^{18} \mathrm{~F}-\mathrm{FDG}$-PET; pancreatectomy

Received: October 05, $2017 \quad$ Accepted: November 14, $2017 \quad$ Published: January 03, 2018

Copyright: Park et al. This is an open-access article distributed under the terms of the Creative Commons Attribution License 3.0 (CC BY

3.0), which permits unrestricted use, distribution, and reproduction in any medium, provided the original author and source are credited.

\section{ABSTRACT}

Objective: We aimed to investigate the metabolic characteristics of Solid pseudopapillary neoplasms (SPNs) in relation signal intensities on ${ }^{18}$ F-FDG PET scans.

Summary Background Data: SPNs of the pancreas commonly show high uptake of 18F-FDG. However, the metabolic characteristics underlying the high ${ }^{18}$ F-FDG uptake in SPNs are not well characterized.

Materials and Methods: mRNA expressions for glucose metabolism were analyzed in five SPNs, five pancreatic ductal adenocarcinomas (PCAs), and paired normal pancreatic tissues. Among the proteins involved in glucose metabolism, the expressions of five proteins (GLUT1, HK1, PFKM, ENO2, and PKM2) were evaluated in 36 SPNs by immunohistochemistry. Clinical patterns of SPN on PET scans were classified according to the proportion of ${ }^{18} \mathrm{~F}-\mathrm{FDG}$ uptake within the whole tumor volume (hot: $\geq \mathbf{7 0 \%}$, mixed: $\mathbf{3 0} \leq<\mathbf{7 0}$, and defective: $<30 \%$ ). PET-based parameters, including maximum standardized uptake value $\left(S U V_{\max }\right)$ and metabolic tumor volume $\left(\mathrm{TMV}_{2.5}\right)$, were evaluated.

Results: Hot $(n=19)$, mixed $(n=5)$, and defective $(n=12){ }^{18}$ F-FDG uptake patterns were noted in the $\mathbf{3 6}$ patients. Radiologic tumor size and SUV max $_{\text {differed }}$ significantly according to these patterns (ANOVA, $p<0.05$ ). GLUT1, HK1, PFKM, ENO2, and PKM2 were highly expressed in SPNs at both the mRNA and protein levels. Defective type SPNs showed lower expression of HK1 $(p=0.014), \operatorname{PKM} 2(p=0.028)$, and Ki-67 $(p=0.070)$ with frequent intra-tumoral necrosis $(p=0.007)$. High Ki-67

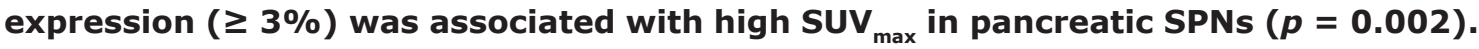

Conclusions: SPN cells harbor an active molecular capacity for increased glucose metabolism. Especially, defective type SPNs were associated with low metabolic activity and related to low $\mathrm{Ki}-67$ index.

\section{INTRODUCTION}

Solid pseudopapillary neoplasm (SPN) is a very rare pathologic condition of the pancreas. It accounts for only
$1-3 \%$ of all exocrine pancreatic tumors and $6-12 \%$ of all cystic tumors of the pancreas. In spite of its clinical rarity, clinical reports related with differential diagnosis have increased. In fact, a recent Korean nationwide survey of 
cystic neoplasms of the exocrine pancreas [1] found SPNs to rank as the third most common cystic neoplasm of the pancreas (18.3\%, 195 out of 1064 patients), indicating that pancreatic SPNs may not be uncommon in Korea. Reportedly, approximately 10 to $15 \%$ of cases of SPN are malignant, although complete surgical resection of these tumors can promise long-term survival, even in cases of distant metastasis and peritoneal seeding [2-5]. Although additional genetic analysis is required to identify the exact mechanism for tumorigenesis of SPN, studies have recently found that SPNs exhibit $\beta$-catenin gene mutation $[6,7]$. Diffuse cytoplasmic and nuclear localization of $\beta$-catenin is indeed found in SPN. $\beta$-catenin functions as a downstream transcriptional co-activator of the Wnt signaling pathway; therefore, the Wnt signal pathway is thought to play an important role in the tumorigenesis of SPN.

According to clinical experience and reports in the literature, [8-11]. SPNs commonly exhibit high uptake of ${ }^{18} \mathrm{~F}-\mathrm{FDG}$. However, investigations into the mechanisms for ${ }^{18}$ F-FDG uptake in SPNs are scarce. In 2006, Sato, et al. [12] reported poor expression of GLUT1 and moderate expression of HK2 on immunohistochemistry staining in SPN cells, suggesting that FDG accumulation might be related to tumor cell density and rich mitochondria, based on the analysis of two cases. Notwithstanding, ${ }^{18}$ F-FDG PET scan can reveal the metabolic and biologic activity of tumors, and it only focuses on the initial steps of glucose metabolism, such as glucose transport and hexokinase activity, not the entire pathway of glucose metabolism. Therefore, in this study, we investigated the metabolic characteristics of SPNs of the pancreas in relation signal intensities on ${ }^{18} \mathrm{~F}$-FDG PET scans.

\section{RESULTS}

\section{Clinical patterns of ${ }^{18} \mathrm{~F}$-FDG uptake in SPNs}

36 patients with SPNs underwent an ${ }^{18}$ FDG-PET scan during preoperative evaluation. 35 patients $(97.2 \%)$ were female and only one was male, with an overall mean age of $34.8 \pm 11.2$ years. Radiologic tumor size was 4.8 $\pm 2.8 \mathrm{~cm}$ in maximum diameter. PET-related parameters were calculated in all SPNs. SUV ${ }_{\max }$ was $5.5 \pm 4.1$ (g/ $\left.\mathrm{cm}^{3}\right)$, and $\mathrm{MTV}_{2.5}$ was $31.5 \pm 59.6\left(\mathrm{~cm}^{3}\right)$. A hot uptake pattern was identified in 19 patients (Figure 1A, and 1B, $52.8 \%$ ), mixed pattern in five (Figure 1C, and 1D, 13.8\%), and defective pattern in 12 patients (Figure 1E, and $1 \mathrm{~F}$, $33.4 \%)$.

When analyzing clinical patterns of ${ }^{18} \mathrm{FDG}$-uptake with radiologic tumor size and PET-parameters, radiologic tumor size $(p=0.002)$ and $\mathrm{SUV}_{\text {max }}(p=0,001)$ differed significantly according to pattern of ${ }^{18} \mathrm{~F}-\mathrm{FDG}$ uptake in SPNs. Mixed type SPNs were larger in size and showed higher intensities of ${ }^{18}$ FDG uptake (ANOVA, $p<0.05$, Figure $1 \mathrm{G}, 1 \mathrm{~F}$ and Supplementary Table 1).

\section{Gene expression profiles for glucose metabolism in SPNs}

We analyzed and compared the expression of genes involved in glucose metabolism and $\beta$-catenin in SPNs, normal pancreas and PCA specimens. These data were selectively obtained from our previous microarray study [13]. Typically, greater over-expression of $\beta$-catenin was noted in SPNs, compared with PCA (4.3-fold in SPN compared to normal pancreatic tissue, $p=0.003$, and 1.6-fold in PCA, $p>0.05$; Figure 2A and Supplementary Table 2). Expression of GLUT1 was significantly higher in PCAs than SPNs (2.2-fold in SPN, $p<0.05$, 11.0-fold in PCA, $\mathrm{p}<0.01)$. However, GLUT12 was significantly higher in SPNs than PCA (in SPN, 19.8-fold, $p<0.001$ and in PCAs, 2.1-fold, $p=0.067)$. GLUT14 was highly expressed in both SPNs and PCAs of the pancreas, compared with normal pancreatic tissue $(0.001<p<0.05)$. Glucose transporter, GLUT6, was expressed in both SPNs and PCAs, compared to normal pancreatic tissue, with significance $(0.01<p<0.05)$ (Supplementary Table 2$)$.

Hexokinases were also highly expressed in both SPNs and PCAs, although HK1 was strongly over-expressed in SPNs, compared with PCAs (7.0-fold in SPN, $p<0.01$ and 3.7-fold in PCA, $p<0.01$ ). Meanwhile, expression of HK2 in SPNs was similar to that in normal pancreatic tissue (1.9-fold, $p>0.05$ ), and higher in PCAs (4.8-fold, $p$ $<0.01$ ). In addition, PFKM (phosphofructokinase, muscle), ENO2 (enolase-2), and PKM2 (pyruvate kinase) were also significantly overexpressed in SPNs. Together, these data suggest that SPNs have a sufficient molecular apparatus from which to active glucose metabolism (Supplementary Table 2 and Supplementary Figure 2).

Interestingly, expression of PDHB (pyruvate dehydrogenase) was similar in SPNs (1.0-fold, $p>0.1)$ and PCAs (-1.1-fold, $p>0.1)$, compared to normal pancreatic tissue. Meanwhile, LDHA (lactate dehydrogenase) was significantly overexpressed in PCAs, compared with normal pancreatic tissue (, 2.9-fold, $p<0.01)$, in contrast to SPNs in which expression of LDHA was lower than that in normal pancreatic tissue, but there were no statistical difference. (-1.1-fold, $p>0.1$ ) (Supplementary Table 2 and Supplementary Figure 2).

\section{Protein expression for glucose metabolism in SPNs}

We performed western blot analysis for the expressions of GLUT1, HK1, PFKM, ENO2, and PKM2 in the same specimens used in the microarray analysis. As shown in gene expression data, overexpression of HK1, ENO2, and PKM2 were also noted at the protein level (Figure 2B). Expression of HK1 and ENO2 were apparently unique to SPNs, compared to PCAs. Greater expression of the GLUT1 gene in SPNs was noted (2.2fold, $p=0.012$ ), compared with PCA (11-fold, $p<0.001$, 
Figure 2A and Supplementary Table 2); however, GLUT1 expression at the protein level was similar between SPNs and PCAs. The expressions of PDHB and LDHA in SPNs were similar to those in normal pancreatic tissue, although expression of LDHA was greater in PCAs, as observed in the gene expression data.

\section{Immunohistochemistry for glucose metabolism in SPNs}

Most of the glucose metabolism-related genes found in the DNA microarray analysis were confirmed in immunohistochemical analysis of resected SPNs (Supplementary Figure 1 and Table 1). GLUT1 was found to be expressed in 31 patients $(86.1 \%$, Supplementary Figure 1A). Greater expression of ENO2 was also noted compared with normal acinar cells (Supplementary Figure
1C). LDHA and PDHB expression was also observed in all patients, which seemed similar to or less than that in normal acinar cells (Supplementary Figure 1E, and $1 F)$. However, HK1 was significantly expressed in the 26 patients (Supplementary Figure 1B), and PKM2 was clearly expressed in 20 patients $(76.9 \%$, Supplementary Figure 1D). All SPNs showed very low proliferative index in Ki-67 (Supplementary Figure 1G, and 1H). Ki67 expression less than 3\% (range, $0-5 \%$ ) was found in almost all patients (94.4\%), and 23 SPNs (63.8\%) showed Ki-67 expression less than 1\%. Immunohistochemistry for identifying glucose metabolism-related genes was also performed in 5 patients with PCAs (Supplementary Table 3). As expected, GLUT1 was expressed in all 5 patients; however, HK1 was rarely expressed and no ENO2 expression was noted in PCAs. Compared to SPNs, PKM2 and LDHA were more strongly expressed in PCAs.
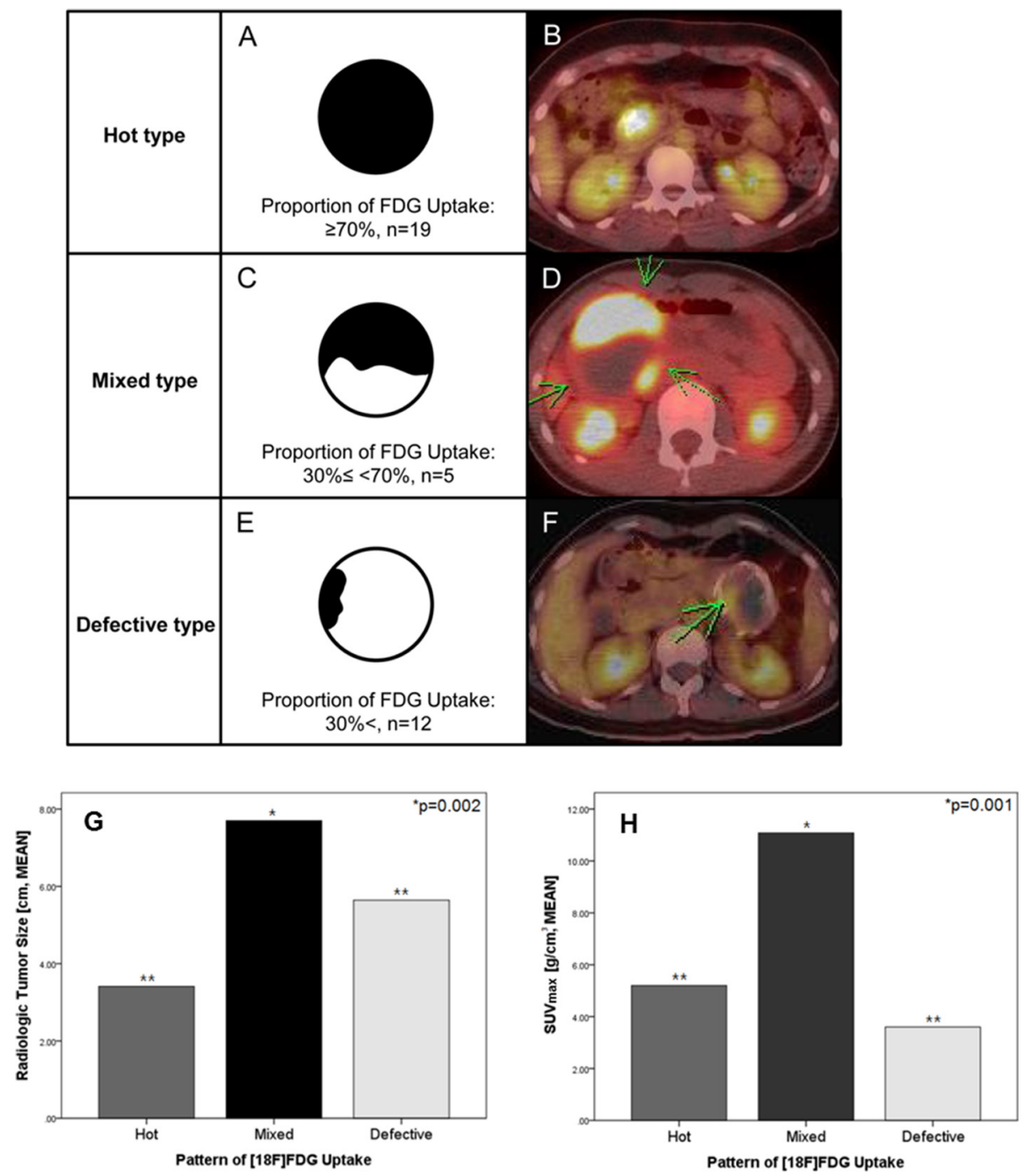

Figure 1: Clinical patterns of 18FDG-uptake in SPN of the pancreas. Clinical patterns of ${ }^{18} \mathrm{~F}-\mathrm{FDG}$ uptake in SPNs were categorized according to the proportion of ${ }^{18} \mathrm{~F}-\mathrm{FDG}$ uptake over the whole tumor volume (Hot type: $\geq 70 \%$ (A, B), Mixed type: $30 \% \leq<$ $70 \%(\mathbf{C}, \mathbf{D})$, and Defective type: $<30 \%(\mathbf{E}, \mathbf{F}))$. When correlating the clinical patterns of ${ }^{18} \mathrm{FDG}$-uptake with radiologic tumor size and PET-parameters, radiologic tumor size $(\mathbf{G}, p=0.002)$ and $\mathrm{SUV}_{\text {max }}(\mathbf{H}, p=0,001)$ were significant different according to pattern of ${ }^{18} \mathrm{~F}-\mathrm{FDG}$ uptake in SPNs of the pancreas. Mixed type of SPN was shown to be large in size with high intensity of ${ }^{18}$ FDG uptake. ${ }^{* *}$ ANOVA, $\left.p>0.05\right)$. 


\section{Correlation between PET-based parameters and glucose metabolism-related gene expressions in SPN}

GLUT1 expression was not associated with ${ }^{18}$ FDG-uptake intensity in SPNs. There was no difference in GLUT1 expression according to pattern of ${ }^{18}$ FDG-uptake ( $p=0.646$, Supplementary Table 4). However, expression of HK1 $(p=0.014)$ and PKM2 $(p=0.028)$ were found differ according to pattern of ${ }^{18}$ FDG-uptake. Expression of HK1 and PKM2 decreased in defective pattern, comparing with hot and mixed patterns (Supplementary Figure 3A, and 3B). Intratumoral necrosis was significantly associated with defective type SPNs ( $p=0.007$, Supplementary Figure $3 \mathrm{C}$, and Supplementary Table 4). In addition, there was a significant association between intra-tumoral necrosis and $\mathrm{Ki}-67$ index ( $p=0.017$, Table 2$)$, indirectly suggesting SPNs with intra-tumoral necrosis are related with a lower Ki-67 index. Therefore, defective type had a tendency to show lower proliferation power, compared with hot and mixed SPNs ( $p=0.07$, Supplementary Figure 3D). Also, SPNs with high ${ }^{18} \mathrm{~F}-\mathrm{FDG}$ intensity $\left(\mathrm{SUV}_{\max }\right.$ ) showed higher Ki-67 index (ANOVA, $p=$ 0.002, Figure 3).

\section{Correlation between expression of glucose metabolism-related genes, patterns of FDG uptake, and microscopic malignant feature of SPNs}

Seven (19.4\%) out of 36 patients were found to have microscopic malignant features in resected SPNs. Microscopic malignant features included capsule invasion, perineural invasion, vascular invasion, infiltrative to pancreatic tissue, and peripancreatic tissue invasion. PETbased parameters $\left(\mathrm{SUV}_{\max }\right.$ and $\left.\mathrm{MTV}_{2.5}\right)$ were similar between SPN groups with microscopic benign and microscopic malignant features $(p>0.05$, Table 3$)$. There were no relationships between expression of GLUT1, HK1, and PKM2, as well as Ki-67 index, and microscopic malignant features $(p>0.05)$. However, defective type SPNs tended to be associated with benign-looking SPNs, compared with hot and mixed type SPNs, although this difference did not reach statistical significance ( $p=0.070$, Table 4$)$.

\section{DISCUSSION}

To date, SPN characteristics on ${ }^{18} \mathrm{~F}$-FDG PET or PET/ $\mathrm{CT}$ have rarely been reported. According to the literature, the intensity of ${ }^{18}$ FDG-uptake in SPNs varies widely. In some
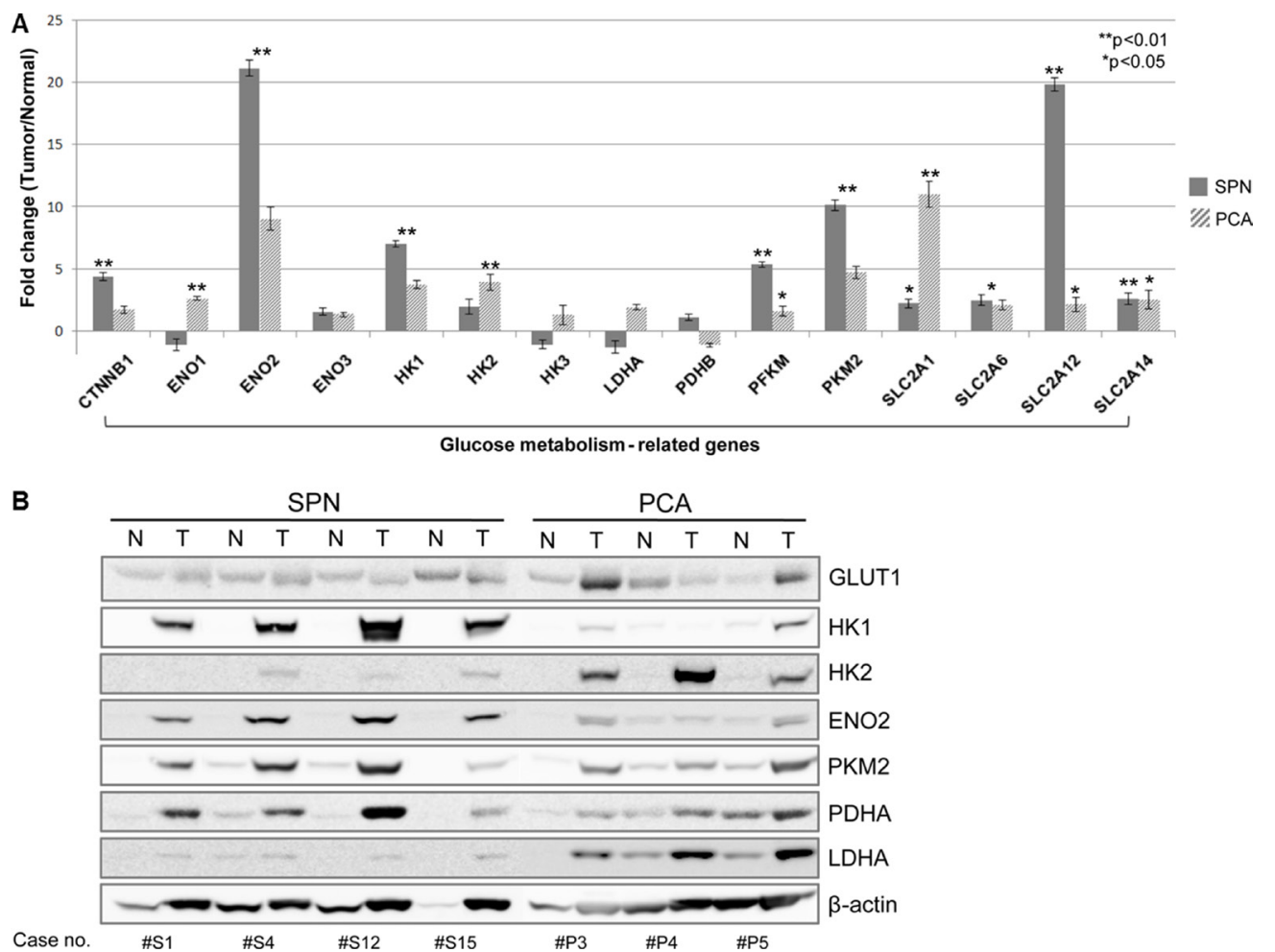

Figure 2: mRNA expression profiles and protein levels of genes for glucose metabolism in SPN. Gene expression profiles of SPNs showed increased expression of genes involved in glucose metabolism. Note that LDHA is highly expressed in PCA comparing to normal pancreatic tissue, but it slightly decreased in SPN without statistical significance (A) See also Supplementary 2). Protein levels of HK1, ENO2, and PKM2 were overexpressed. Expression of HK1 and ENO2 were upregulated specifically in SPNs, compared to PCAs. Meanwhile, protein expression of GLUT1 was similar between SPNs and PCAs. The expressions of PDHB and LDHA in SPNs were similar to those in normal pancreatic tissue, although expression of LDHA was greater in PCAs (B). SPN, solid pseudopapillary tumor; PCA, pancreatic ductal adenocarcinoma. 
reports, $[14,15]$ SPNs with mild or no ${ }^{18} \mathrm{~F}$-FDG uptake have been presented, while others have described SPNs with intense ${ }^{18}$ F-FGD uptake $[8,9,12,16,17]$. With accumulating clinical experience in SPN, application of PET scans to SPNs of the pancreas has expanded to testing its capacity to differentiate SPNs from other malignant tumors, such as pancreatic cancer or neuroendocrine tumor, [10, 11, 18, 19] in addition to its clinical usefulness in staging and treatment planning $[16,17]$. Although several reports of high ${ }^{18} \mathrm{~F}-\mathrm{FDG}$ uptake in SPN have been published, research on the metabolic mechanisms of SPNs is rarely reported. It is thought that any tumor will cause the alterations of the glucose metabolism, then glucose metabolism should not be the specific for SPNs. However, there was no study to investigate glucose metabolic alteration in SPNs to confirm this assumption. The exact mechanisms involved in glucose uptake by SPNs are still unknown. Therefore, we primarily tried to overview the landscape of altered glucose metabolism in resected SPNs of the pancreas, especially comparing with pancreatic cancer.

In present study, based on our previous research of characterizing gene expression profiles for SPNs, [13]. we investigated the molecular mechanisms of ${ }^{18} \mathrm{~F}-\mathrm{FDG}$ uptake in SPNs. To the best of our knowledge, the present study is the first to report on a molecular mechanism of glucose metabolism in SPNs. According to our data, SPNs show a distinct molecular apparatus for glucose uptake and for glycolysis to form pyruvate. We found that GLUT1, GLUT6, GLUT12, and GLUT14 are highly expressed in SPNs. GLUT1, known as a principal glucose transporter in tumors, was expressed even at the protein level. In addition, enzymes involved in glycolysis, such as HK1, ENO2, and PKM2, were also overexpressed, and this was confirmed in both western blot and immunohistochemistry experiments. These molecular profiles strongly suggest that neoplastic cells of SPN possess an increased capacity for glucose metabolism, which is observable in the appearance of SPNs on ${ }^{18} \mathrm{~F}-\mathrm{FDG}$-PET scans.

This study also attempted to categorize clinical patterns of ${ }^{18} \mathrm{~F}$-FDG uptake in SPNs. We previously classified patterns of ${ }^{18} \mathrm{~F}-\mathrm{FDG}$ uptake in SPNs into five categories [20]. In addition, we also tried to categorize SPNs according to different ${ }^{18} \mathrm{~F}$-FDG-uptake proportion such as, < 10\%, 10\%-30\%, 30-50\%, 50-70\%. 70$90 \%$, and $90 \%<$, and the similar relationship with the present results were founded (data not shown). These classification system looks very specific, but it was found that these categories were complex and confused to apply in clinical practice. So, we slightly modified previous five categories simply into Hot uptake (previously belong to type I and type II), Mixed (previously belong to type III), and Defective type (previously belong to type IV and V) in this study based on the proportions of ${ }^{18} \mathrm{~F}$-FDG uptake within the whole tumor.

In the present study, we found that intra-tumoral necrosis was found to be associated with low Ki-67 index (Table 2), and defective type SPNs were related to intra-tumoral necrosis and a lower $\mathrm{Ki}-67$ index (Supplementary Figure 3C, and 3D). These observations indirectly suggest that defective type SPNs may be less likely to progress. Defective type SPNs, resulting from

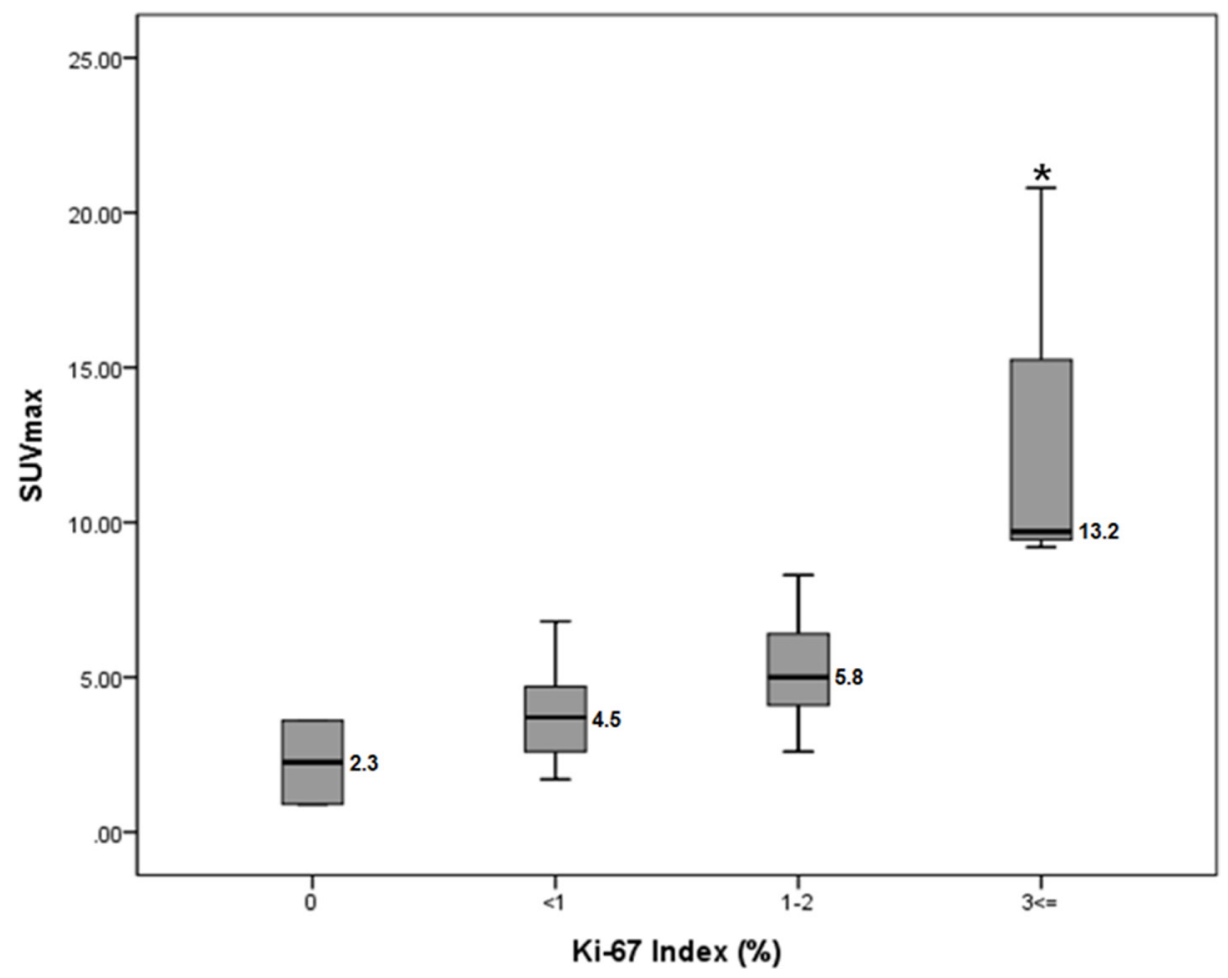

Figure 3: $\mathrm{SUV}_{\max }$ values according to Ki-67 Index. SPNs with high ${ }^{18} \mathrm{~F}-\mathrm{FDG}$ intensity $\left(\mathrm{SUV}_{\max }\right)$ showed higher Ki-67 index (ANOVA). ${ }^{*} p=0.002$, a number beside the box shows the mean value of $\mathrm{SUV}_{\max }$ 
intra-tumoral hemorrhagic necrosis due to weakened cell-to-cell adhesion, [21] can cause chronic hypoxia and ischemic necrosis of viable solid portions of the tumor. If the tumors are not clinically detected, even at this stage, and/or not treated immediately, a totally necrotic tumor of the pancreas might be found. Recently, we proposed that marginally calcified, totally necrotic pancreatic tumors might comprise a subset of SPNs with near total necrosis [22] (perhaps defective type $\mathrm{SPN}$ ), and the present observation may support this hypothesis. It is also interesting to note that the clinical patterns of ${ }^{18} \mathrm{~F}$-FDG uptake in SPNs apparently represent the metabolic activity of SPN. Expression of HK1 and PKM2 were closely correlated with patterns of ${ }^{18} \mathrm{~F}-\mathrm{FDG}$ uptake (Supplementary Figure 3A, 3B and Supplementary Table 4). More frequent expression of HK1 and PKM2 were noted in hot and mixed pattern, compared to the defective pattern. Pyruvate kinase is the last rate-limiting enzyme in glycolysis, and catalyzes the conversion of phosphoenolpyruvate and ADP into pyruvate and ATP. PKM2 is known to be expressed predominantly in tumor cells, and is important for cancer metabolism and tumor growth. A previous study showed that PKM2 expression is involved in early tumorigenesis [23]. and that increases in PKM2 levels are correlated with tumor size and stage [24]. HK catalyzes the conversion of glucose to glucose-6phosphate, the first and rate-limiting step in the glycolytic pathway. Usually, HK2 is regarded as a principle enzyme in cancer metabolism; however, our study suggests that HK1 is the main enzyme converting glucose to glucose6-phosphate in SPNs. Nevertheless, the exact role of HK1 and PKM2 in the tumorigenesis of SPN remains to be investigated further (Supplementary Figure 2).
We also found that SPNs with high Ki-67 index are related with high $\mathrm{SUV}_{\max }$ (Figure 7). Tumor size and PETrelated parameters, such as $\mathrm{SUV}_{\text {max }}$, were larger and higher in mixed type SPNs (Figure 2 and Supplementary Table 1), suggesting mixed type SPNs may be a biologically active tumor with higher Ki-67 index. Due to the limited number of mixed type SPNs in our data set ( $n=5$ patients), it would have been difficult for us to definitively examine this potential relationship; however, this observation is thought to be very important, because Ki-67 index and tumor proliferation have been previously reported to associated with aggressive biological behavior of SPNs [25-27]. Recently, Yu, et al. [28] also demonstrated that positive immunoreactivity for Ki-67 may predict the malignant potential and poor outcomes of SPN. Nakagohri, et al. [14] reported that most SPNs (5 out of 6 tumors) show strong accumulation of FDG on PET scans, and showed that SPNs with high FDG uptake were related to microscopic venous and perineural invasion. Conversely, the tumors without intense FDG uptake had neither microscopic venous invasion nor nerve invasion, suggesting a potential relationship between SUV and histological malignancy. In our data, we observed no relationship between malignant microscopic features in resected SPNs and GLUT1, HK1, intratumoral necrosis, and Ki-67 index. However, patterns of ${ }^{18}$ F-FDG uptake in SPNs were found to be related to microscopic malignant features of SPN with marginal significance $(p=0.070$, Table 4$)$.

Therefore, clinically, it can be recommended that SPT with high $\mathrm{SUV}_{\text {max }}$ (for example, hot uptake and mixed type) should be aggressively treated from the metabolomics point of view, because PET-based parameter, especially high $\mathrm{SUV}_{\max }$ was related to

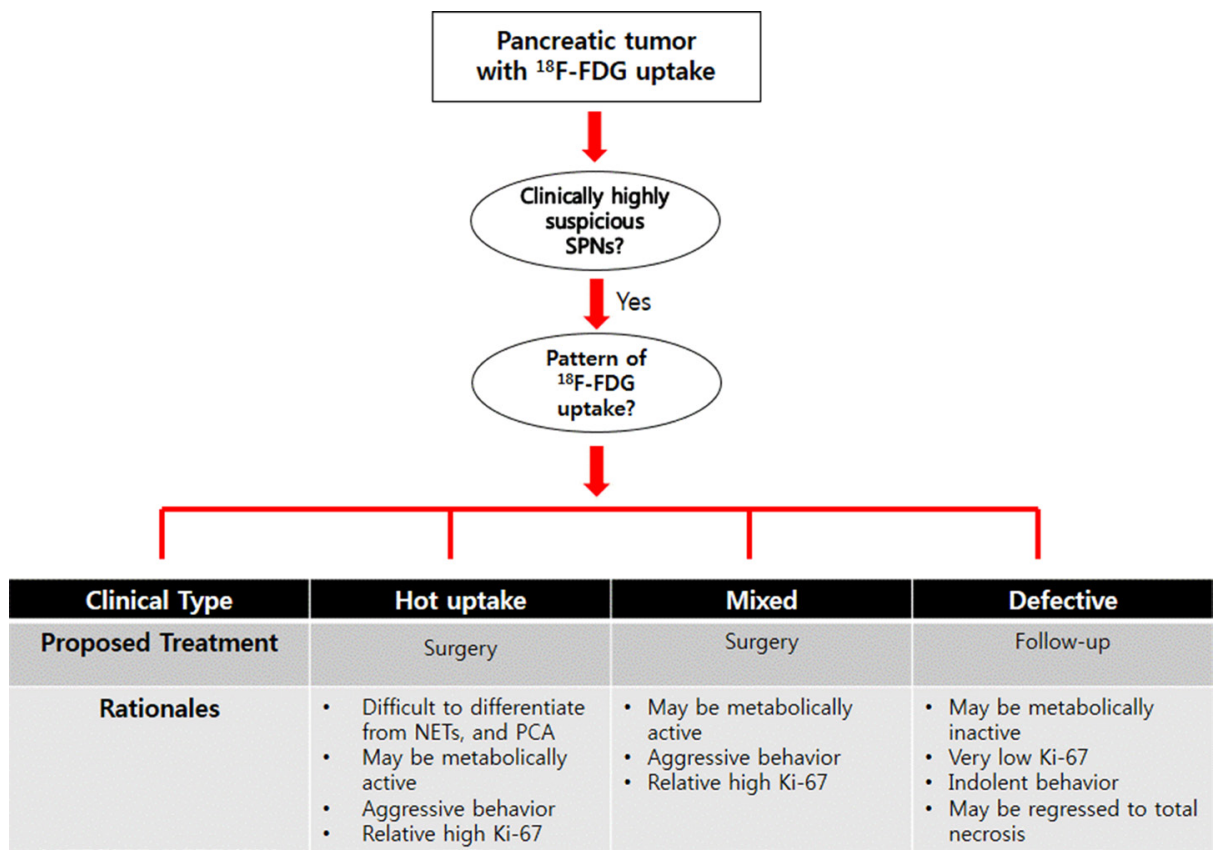

Figure 4: Proposing surgical strategy for SPTs of the pancreas based on the pattern of ${ }^{18}$ F-FDG uptake. Surgical decisionmaking can be specified according to clinical pattern of ${ }^{18} \mathrm{~F}-\mathrm{FDG}$ uptake in patients with SPN. 


\begin{tabular}{|c|c|c|c|c|c|c|c|c|c|c|}
\hline Case\# & Gender & Age & $\begin{array}{c}\text { Tumor } \\
\text { Size }(\mathrm{cm})\end{array}$ & $\begin{array}{l}{ }^{18} \text { FDG- } \\
\text { uptake } \\
\text { pattern }\end{array}$ & GLUT1 & HK1 & ENO2 & PKM2 & Ki-67 & Necrosis \\
\hline 1 & Female & 25 & 5.6 & Hot & + & + & + & $+/-$ & $<1 \%$ & - \\
\hline 2 & Female & 46 & 5 & Defective & - & ++ & + & + & $<1 \%$ & - \\
\hline 3 & Female & 38 & 3.5 & Mixed & + & ++ & + & N/A & $<1 \%$ & + \\
\hline 4 & Female & 28 & 5 & Defective & + & $\mathrm{N} / \mathrm{A}$ & + & N/A & $<1 \%$ & + \\
\hline 5 & Female & 28 & 6.8 & Hot & + & N/A & + & $\mathrm{N} / \mathrm{A}$ & $<1 \%$ & + \\
\hline 6 & Female & 24 & 8.2 & Hot & + & ++ & + & + & $<1 \%$ & - \\
\hline 7 & Female & 32 & 1.7 & Hot & + & ++ & + & + & $1-2 \%$ & - \\
\hline 8 & Female & 25 & 4.9 & Defective & + & ++ & + & + & $1-2 \%$ & - \\
\hline 9 & Female & 31 & 5.3 & Defective & + & $\mathrm{N} / \mathrm{A}$ & + & $\mathrm{N} / \mathrm{A}$ & $0 \%$ & + \\
\hline 10 & Female & 46 & 6.5 & Defective & + & N/A & + & $\mathrm{N} / \mathrm{A}$ & $0 \%$ & + \\
\hline 11 & Female & 45 & 2.3 & Mixed & + & ++ & + & + & $<1 \%$ & - \\
\hline 12 & Female & 12 & 10.1 & Mixed & + & ++ & + & + & $3 \% \leq$ & - \\
\hline 13 & Female & 25 & 12.3 & Hot & + & N/A & + & N/A & $1-2 \%$ & + \\
\hline 14 & Female & 35 & 1.3 & Hot & + & ++ & + & + & $1-2 \%$ & - \\
\hline 15 & Female & 24 & 5.7 & Hot & + & ++ & + & + & $<1 \%$ & - \\
\hline 16 & Female & 62 & 1.5 & Hot & + & ++ & + & + & $<1 \%$ & - \\
\hline 17 & Female & 35 & 1.6 & Mixed & + & ++ & + & + & $<1 \%$ & - \\
\hline 18 & Female & 14 & 6.9 & Hot & - & ++ & + & $+/-$ & $3 \% \leq$ & - \\
\hline 19 & Female & 19 & 3.5 & Mixed & - & ++ & + & + & $1-2 \%$ & - \\
\hline 20 & Female & 47 & 4.2 & Hot & + & ++ & + & + & $<1 \%$ & - \\
\hline 21 & Male & 30 & 3.8 & Hot & + & ++ & + & + & $<1 \%$ & - \\
\hline 22 & Female & 48 & 1.5 & Defective & + & ++ & + & + & $<1 \%$ & - \\
\hline 23 & Female & 41 & 2.5 & Defective & + & N/A & + & N/A & $<1 \%$ & + \\
\hline 24 & Female & 38 & 6.5 & Hot & + & N/A & + & N/A & $<1 \%$ & + \\
\hline 25 & Female & 46 & 2 & Defective & + & ++ & + & + & $1-2 \%$ & - \\
\hline 26 & Female & 28 & 8 & Defective & + & + & + & $+/-$ & $3 \% \leq$ & - \\
\hline 27 & Female & 16 & 7 & Hot & + & $\mathrm{N} / \mathrm{A}$ & + & $\mathrm{N} / \mathrm{A}$ & $<1 \%$ & - \\
\hline 28 & Female & 45 & 3 & - & - & N/A & + & $+/-$ & $<1 \%$ & + \\
\hline 29 & Female & 43 & 5.2 & Defective & - & $\mathrm{N} / \mathrm{A}$ & + & $+/-$ & $<1 \%$ & + \\
\hline 30 & Female & 22 & 8.5 & Hot & + & + & + & + & $1-2 \%$ & - \\
\hline 31 & Female & 40 & 8.3 & Hot & + & ++ & + & + & $1-2 \%$ & - \\
\hline 32 & Female & 35 & 1.6 & Hot & + & ++ & + & + & $<1 \%$ & - \\
\hline 33 & Female & 38 & 2 & Hot & + & ++ & + & N/A & $1-2 \%$ & - \\
\hline 34 & Female & 40 & 2.7 & Hot & + & + & + & $\mathrm{N} / \mathrm{A}$ & $<1 \%$ & - \\
\hline 35 & Female & 42 & 1.5 & Hot & + & ++ & + & + & $1-2 \%$ & - \\
\hline 36 & Female & 23 & 5 & Hot & + & ++ & + & $+/-$ & $<1 \%$ & - \\
\hline
\end{tabular}

N/A; not available due to necrosis, immunohistochemistry score (-; negative, +/-; weak, +; moderate, ++ ; strong)

increased Ki-67 index. In addition, small hot-uptake type of SPT should be considered for resection because it is difficult to differentiate from other malignant tumors of the pancreas, such as pancreatic cancer, and neuroendocrine tumor, when the tumor did not show typical radiologic characteristics of SPNs of the pancreas $[11,18]$. On the other hand, defective type of SPNs was associated with low expression of HK1/ PKM2, intratumoral necrosis, and tended to have lower proliferation with low metabolic capacity (Figure 1H, 
Table 2: Correlation between intratumoral necrosis and Ki-67 index

\begin{tabular}{lcccc}
\hline & & Necrosis & \multirow{2}{*}{$\boldsymbol{P}$-value } \\
\cline { 2 - 4 } Ki-67 (\%) & 0 & Negative & Positive & \\
& $<1$ & 0 & 2 & 0.017 \\
& $1-2$ & 9 & 7 & \\
& $3 \leq$ & 3 & 1 & 0 \\
\hline
\end{tabular}

Table 3: Correlation between PET-based parameters and microscopic malignant features of SPNs

\begin{tabular}{cccc}
\hline & \multicolumn{2}{c}{ Microscopic malignant features } & \multirow{2}{*}{$\boldsymbol{P}$-value } \\
\cline { 2 - 3 } & Absent $(\boldsymbol{N}=\mathbf{2 9})$ & Present $(\boldsymbol{N}=\mathbf{7})$ & 0.299 \\
$\mathrm{SUV}_{\max }$ & $5.1 \pm 4.0$ & $6.9 \pm 4.5$ & 0.284 \\
$\mathrm{MTV}_{2.5}$ & $23.3 \pm 47.2$ & $65.3 \pm 92.8$ & 0.828 \\
\hline Tumor size & $4.9 \pm 3.2$ & $5.1 \pm 2.9$ & \\
\hline
\end{tabular}

and Figure 3), suggesting this metabolic type of SPNs might be less progressive and would follow indolent clinical course. Considering most patients with SPNs are young female patients with active social activity, surgery for SPTs with defective type of ${ }^{18} \mathrm{~F}-\mathrm{FDG}$ uptake could be reserved according to patient's social activity, and physical conditions, because it cannot be denied that pancreatectomy is related to high rate of postoperative morbidity with potential mortality in spite of improved perioperative management [29] (Figure 4).

Based on the current observations, further investigation of the metabolic differences between indolent type SPNs and those with clinically aggressive behavior could prove valuable. What makes indolent SPNs change to take on an aggressive biologic behavior? What differences exist between types of SPN from the view point of metabolism? Why should SPNs show increased glucose metabolism in spite of low proliferation? Is there any specific metabolomics pathway for SPNs? Future basic and clinical research should provide answers to this surgical enigma.

\section{MATERIALS AND METHODS}

\section{Patient selection and clinical data}

The medical records of 36 patients with SPN who underwent preoperative ${ }^{18} \mathrm{~F}$-FDG PET/PET-CT scans and a pancreatectomy were retrospectively reviewed. The clinicopathologic characteristics of the patients, including age, gender, radiologic tumor size, and microscopic malignant features, were investigated. SPN tissue samples from 36 patients were obtained by surgical resection. Among them, five fresh tissues that consisted of more than $70 \%$ tumor cells without previous adjuvant chemo- or radiotherapy were selected for analysis of gene expression profiles. The specimens were obtained from the archives of the Department of Pathology, Yonsei University, Seoul, Korea and from the Liver Cancer Specimen Bank of the National Research Resource Bank Program of the Korea Science and Engineering Foundation under the Ministry of Science and Technology. Authorization for the use of these tissues for research purposes was obtained from the Institutional Review Board of Yonsei University of College of Medicine.

\section{${ }^{18}$ F-FDP PET/ PET-CT protocol}

All ${ }^{18} \mathrm{~F}$-FDG PET/PET-CT scans were performed with a dedicated PET/CT scanner (Discovery STe, GE Healthcare; or Biograph TruePoint 40, Siemens Healthcare). All patients fasted for at least $6 \mathrm{~h}$ prior to the PET/CT scan. A dose of approximately $5.5 \mathrm{MBq} /$ $\mathrm{kg}$ of ${ }^{18} \mathrm{~F}$-FDG was intravenously injected $60 \mathrm{~min}$ before imaging. First, CT scans were performed at $30 \mathrm{~mA}$ and 130 $\mathrm{kVp}$ with the Discovery STe scanner or at $36 \mathrm{~mA}$ and 120 $\mathrm{kVp}$ with the Biograph TruePoint scanner without contrastenhancement. After the CT scan was complete, a PET scan was performed from the neck to the proximal thigh, with an acquisition time of $3 \mathrm{~min}$ per bed position in a 3D mode. PET images were reconstructed using ordered subset expectation maximization with attenuation correction.

\section{Image evaluation and PET-based parameters}

${ }^{18} \mathrm{~F}-\mathrm{FDG} \mathrm{PET} / \mathrm{CT}$ images were reviewed by two nuclear medicine physicians (Yun M, and Cho A) using 
Table 4: Correlation between staining intensity of glucose metabolism-related genes and microscopic malignant features of SPNs

\begin{tabular}{|c|c|c|c|c|}
\hline & & \multicolumn{2}{|c|}{ Microscopic malignant features } & \multirow[b]{2}{*}{$P$-value } \\
\hline & & Absent $(N=29)$ & $\begin{array}{l}\text { Present } \\
(N=7)\end{array}$ & \\
\hline \multirow[t]{2}{*}{ GLUT1 } & - & 4 & 1 & \multirow{2}{*}{1.000} \\
\hline & + & 25 & 6 & \\
\hline \multirow[t]{2}{*}{ HK1 ${ }^{*}$} & + & 3 & 1 & \multirow{2}{*}{1.000} \\
\hline & ++ & 17 & 5 & \\
\hline \multirow[t]{2}{*}{ PKM2* } & $+/-$ & 6 & 0 & \multirow{2}{*}{0.280} \\
\hline & + & 14 & 6 & \\
\hline \multirow[t]{2}{*}{ Necrosis } & - & 20 & 6 & \multirow{2}{*}{0.645} \\
\hline & + & 9 & 1 & \\
\hline \multirow[t]{4}{*}{ Ki-67 } & 0 & 2 & 0 & \multirow{4}{*}{0.781} \\
\hline & $<1$ & 15 & 6 & \\
\hline & $1-2$ & 10 & - & \\
\hline & $3 \leq$ & 2 & 1 & \\
\hline \multirow{2}{*}{$\begin{array}{c}\text { Clinical pattern }{ }^{18} \text { FDG-uptake } \\
\text { Defective }\end{array}$} & Hot + Mixed & 17 & 7 & \multirow{2}{*}{0.070} \\
\hline & 12 & 0 & & \\
\hline
\end{tabular}

* 10 cases were not included due to poor staining.

an Advantage Workstation 4.4 (GE Medical Systems). Maximum standardized uptake value $\left(\mathrm{SUV}_{\max }\right)$ and metabolic tumor volume $\left(\mathrm{TMV}_{2.5}\right)$ on PET images were measured using the volume viewer software. Each tumor was examined with a spherical-shaped volume of interest (VOI) that included the entire lesion in the axial, sagittal, and coronal planes. By using $\mathrm{CT}$ images, ${ }^{18} \mathrm{~F}$-FDG uptake by normal organs, such as the bowel, stomach, and liver, was excluded from the VOI. The $\mathrm{SUV}_{\max }$ of the VOI was calculated as (decay-corrected activity/tissue volume)/(injected dose/body weight). $\mathrm{MTV}_{2.5}$ was defined as the total tumor volume with an $\mathrm{SUV} \geq 2.5$. In patients with a $\mathrm{SUV}_{\text {max }}$ of $<2.5, \mathrm{MTV}_{2.5}$ was not measured. In addition, clinical patterns of ${ }^{18} \mathrm{~F}-\mathrm{FDG}$ uptake in SPNs were categorized according to the proportion of ${ }^{18} \mathrm{~F}$-FDG uptake over the whole tumor volume (hot: $\geq 70 \%$, mixed: $30 \% \leq 70 \%$, and defective: $<30 \%$, Figure 1).

\section{mRNA gene expression data preparation and statistical analysis}

Raw data were extracted using the software provided by Illumina Genome Studio v2011.1 (Gene Expression Module v1.9.0). Expression intensities were normalized using quantile normalization techniques. Using the normalized intensities, differentially expressed genes (DEGs) between non-neoplastic pancreatic tissue and pancreatic tumors (SPN, or PCA) were determined using a previously reported integrated statistical method [13]. We selected the expression of genes involved in glucose metabolism in five SPNs, and compared their results to those of normal pancreas and PCA specimens.

\section{Western blot}

Whole lysates from tissue specimens were prepared using passive lysis buffer (Promega). Protein samples were separated by SDS-PAGE and transferred to nitrocellulose membranes. Blots were blocked with Trisbuffered saline and Tween 20 containing 5\% skim milk, and incubated overnight at $4^{\circ} \mathrm{C}$ with primary antibodies against GLUT1 (Alpha Diagnostic), HK1, PKM2, LDHA, PDHA (Cell Signaling) and ENO2 (antibodies-online). After washing, the membranes were incubated with horseradish peroxidase-conjugated secondary antibody (Santa Cruz Biotechnology) for $1 \mathrm{~h}$ at room temperature, washed, and developed with luminol reagent (Santa Cruz Biotechnology).

\section{Immunohistochemistry}

Healthy, available paraffin-embedded tissue blocks for the 36 patients were cut into $4-\mu \mathrm{m}$ sections. Immunohistochemical analysis was performed using a Ventana XT automated stainer (Ventana Corporation) with antibodies against GLUT1 (Alpha Diagnostic), HK1, PKM2, LDHA, PDHA (Cell Signaling), ENO2 (antibodies- 
online), and Ki-67 (Dako). Immunohistochemical results were scored according to staining intensities as follows: -, no staining; +/-, weak staining (faint protein expression); + , moderate staining (definite protein staining in $\leq 30 \%$ of tumor cells); or ++ , strong staining (definite protein expression in $>30 \%$ of tumor cells).

\section{Statistical analysis}

Continuous variables are expressed as mean \pm standard deviation and categorical variables as frequency (\%). ANOVA and Student's $t$-test were used for comparative analysis, while chi-square (Fisher's exact test, or linearto-linear association if necessary) was used for analyzing clinical patterns of FDG uptake and immunohistochemical grades for detecting glucose metabolism-related gene expression. Statistical analyses were performed using SPSS software, version 20.0 for Windows (SPSS Inc.). $P$-values $<$ 0.05 were considered statistically significant.

\section{Abbreviations}

Solid pseudopapillary neoplasm: SPN; Winglessrelated integration site: Wnt; Pancreatic ductal adenocarcinomas: PCA; Glucose transporter 1: GLUT1; Hexokinase2: HK2; Fluorodeoxyglucose positron emission tomography: ${ }^{18} \mathrm{~F}-\mathrm{FDG}$ PET; Computed tomography: CT; Maximum standardized uptake value: $\mathrm{SUV}_{\max }$; Metabolic tumor volume: TMV; Pyruvate kinase muscle isozyme 2: PKM2; Lactate dehydrogenase A: LDHA; Pyruvate dehydrogenase E1 component subunit alpha: PDHA; enolase 2: ENO2.

\section{Author contributions}

MHP \& HKH: designed and conducted experiments, analyzed data, wrote and edited the manuscript equally; MJY: designed and analyzed data; WJL: designed and analyzed data, supervised manuscript; HGK \& CMK: conceived of, planned, designed experiments, analyzed data, wrote and edited manuscript, supervising of procedures equally.

\section{CONFLICTS OF INTEREST}

The authors declare no conflicts of interest.

\section{FUNDING}

This research was supported by a grant from the Korea Health Technology R\&D Project through the Korea Health Industry Development Institute (KHIDI), funded by the Ministry of Health \& Welfare, Republic of Korea (grant number: HI14C1324 and HI16C0257).

\section{REFERENCES}

1. Yoon WJ, Lee JK, Lee KH, Ryu JK, Kim YT, Yoon YB. Cystic neoplasms of the exocrine pancreas: an update of a nationwide survey in Korea. Pancreas. 2008; 37:254-258.

2. Sperti C, Berselli M, Pasquali C, Pastorelli D, Pedrazzoli S. Aggressive behaviour of solid-pseudopapillary tumor of the pancreas in adults: a case report and review of the literature. World J Gastroenterol. 2008; 14:960-965.

3. Takahashi Y, Fukusato T, Aita K, Toida S, Fukushima J, Imamura T, Tanaka F, Amano H, Takada T, Mori S. Solid pseudopapillary tumor of the pancreas with metastases to the lung and liver. Pathol Int. 2005; 55:792-796.

4. Nishihara K, Nagoshi M, Tsuneyoshi M, Yamaguchi K, Hayashi I. Papillary cystic tumors of the pancreas. Assessment of their malignant potential. Cancer. 1993; 71:82-92.

5. Zinner MJ, Shurbaji MS, Cameron JL. Solid and papillary epithelial neoplasms of the pancreas. Surgery. 1990; 108:475-480.

6. Abraham SC, Klimstra DS, Wilentz RE, Yeo CJ, Conlon K, Brennan M, Cameron JL, Wu TT, Hruban RH. Solidpseudopapillary tumors of the pancreas are genetically distinct from pancreatic ductal adenocarcinomas and almost always harbor beta-catenin mutations. Am J Pathol. 2002; 160:1361-1369.

7. Tanaka Y, Kato K, Notohara K, Hojo H, Ijiri R, Miyake T, Nagahara N, Sasaki F, Kitagawa N, Nakatani Y, Kobayashi Y. Frequent beta-catenin mutation and cytoplasmic/nuclear accumulation in pancreatic solid-pseudopapillary neoplasm. Cancer Res. 2001; 61:8401-8404.

8. Dong A, Wang Y, Dong H, Zhang J, Cheng C, Zuo C. FDG $\mathrm{PET} / \mathrm{CT}$ findings of solid pseudopapillary tumor of the pancreas with CT and MRI correlation. Clin Nucl Med. 2013; 38:e118-124.

9. Shimada K, Nakamoto Y, Isoda H, Maetani Y, Yamashita R, Arizono S, Hirokawa Y, Nitta T, Doi R, Haga H, Togashi K. F-18 fluorodeoxyglucose uptake in a solid pseudopapillary tumor of the pancreas mimicking malignancy. Clin Nucl Med. 2008; 33:766-768.

10. Guan ZW, Xu BX, Wang RM, Sun L, Tian JH. Hyperaccumulation of (18)F-FDG in order to differentiate solid pseudopapillary tumors from adenocarcinomas and from neuroendocrine pancreatic tumors and review of the literature. Hell J Nucl Med. 2013; 16:97-102.

11. Kim YI, Kim SK, Paeng JC, Lee HY. Comparison of F-18-FDG PET/CT findings between pancreatic solid pseudopapillary tumor and pancreatic ductal adenocarcinoma. Eur J Radiol. 2014; 83:231-235.

12. Sato M, Takasaka I, Okumura T, Shioyama Y, Kawasaki H, Mise Y, Asato Y, Yoshimi F, Imura J, Nakajima K. High F-18 fluorodeoxyglucose accumulation in solid pseudopapillary tumors of the pancreas. Ann Nucl Med. 2006; 20:431-436. 
13. Park M, Kim M, Hwang D, Park M, Kim WK, Kim SK, Shin J, Park ES, Kang CM, Paik YK, Kim H. Characterization of gene expression and activated signaling pathways in solid-pseudopapillary neoplasm of pancreas. Mod Pathol. 2014; 27:580-593.

14. Nakagohri T, Kinoshita T, Konishi M, Takahashi S, Gotohda N. Surgical outcome of solid pseudopapillary tumor of the pancreas. J Hepatobiliary Pancreat Surg. 2008; 15:318-321.

15. Kang CM, Kim KS, Choi JS, Kim H, Lee WJ, Kim BR. Solid pseudopapillary tumor of the pancreas suggesting malignant potential. Pancreas. 2006; 32:276-280.

16. Lee JK, Tyan YS. Detection of a solid pseudopapillary tumor of the pancreas with F-18 FDG positron emission tomography. Clin Nucl Med. 2005; 30:187-188.

17. Treglia G, Caporale N, Rufini V, Callea F, Locatelli F, Giordano A. Usefulness of 18F-FDG PET/CT in an unusual case of solid-pseudopapillary pancreatic tumor in childhood with aggressive behavior. Clin Nucl Med. 2013; 38:e35-37.

18. Dong A, Dong H, Zhang L, Zuo C. Hypermetabolic lesions of the pancreas on FDG PET/CT. Clin Nucl Med. 2013; 38:e354-366.

19. Choi HJ, Lee JW, Kang B, Song SY, Lee JD, Lee JH. Prognostic significance of volume-based FDG PET/CT parameters in patients with locally advanced pancreatic cancer treated with chemoradiation therapy. Yonsei Med J. 2014; 55:1498-1506.

20. Kang CM, Cho A, Kim H, Chung YE, Hwang HK, Choi $\mathrm{SH}$, Lee WJ. Clinical correlations with FDG PET scan patterns in solid pseudopapillary tumors of the pancreas: still a surgical enigma? Pancreatology. 2014; 14:515-523.

21. Kang CM, Kim HK, Kim H, Choi GH, Kim KS, Choi JS, Lee WJ. Expression of Wnt target genes in solid pseudopapillary tumor of the pancreas: a pilot study. Pancreas. 2009; 38:e53-59.

22. Kang CM, Hwang HK, Kim H, Kim H, Chung YE, Park JS, Yoon DS, Lee WJ. Marginally calcified totally necrotic tumor of the pancreas: a subset of solid pseudopapillary tumor with near-total necrosis? Pancreas. 2013; 42:184-186.

23. Wittwer JA, Robbins D, Wang F, Codarin S, Shen X, Kevil CG, Huang TT, Van Remmen H, Richardson A, Zhao Y. Enhancing mitochondrial respiration suppresses tumor promoter TPA-induced PKM2 expression and cell transformation in skin epidermal JB6 cells. Cancer Prev Res (Phila). 2011; 4:1476-1484.

24. Mazurek S, Boschek CB, Hugo F, Eigenbrodt E. Pyruvate kinase type M2 and its role in tumor growth and spreading. Semin Cancer Biol. 2005; 15:300-308.

25. Tang LH, Aydin H, Brennan MF, Klimstra DS. Clinically aggressive solid pseudopapillary tumors of the pancreas: a report of two cases with components of undifferentiated carcinoma and a comparative clinicopathologic analysis of 34 conventional cases. Am J Surg Pathol. 2005; 29:512-519.

26. Hibi T, Ojima H, Sakamoto Y, Kosuge T, Shimada K, Sano T, Sakamoto M, Kitajima M, Yamasaki S. A solid pseudopapillary tumor arising from the greater omentum followed by multiple metastases with increasing malignant potential. J Gastroenterol. 2006; 41:276-281.

27. Lai HW, Su CH, Li AF, Wu LH, Shyr YM, Chen TH, Wu CW, Lui WY. Malignant solid and pseudopapillary tumor of the pancreas--clinicohistological, immunohistochemical, and flow cytometric evaluation. Hepatogastroenterology. 2006; 53:291-295.

28. Yu P, Cheng X, Du Y, Yang L, Xu Z, Yin W, Zhong Z, Wang $\mathrm{X}, \mathrm{Xu} \mathrm{H}, \mathrm{Hu} \mathrm{C}$. Solid Pseudopapillary Neoplasms of the Pancreas: a 19-Year Multicenter Experience in China. J Gastrointest Surg. 2015; 19:1433-1440.

29. Mezhir JJ. Management of complications following pancreatic resection: an evidence-based approach. J Surg Oncol. 2013; 107:58-66. 